\title{
Lipid Emulsion Has No Effect on the Hemodynamic Cardiovascular Toxicity of Ropivacaine. Study in an Experimental Porcine Model
}

\author{
García S1, López- Menchaca R¹, Melone A1, Varela O', Sevilla R1, Zaballos M1 \\ 1- (Department of Anaesthesia, Hospital Gregorio Marañón (Madrid, Spain)
}

\section{BACKGROUND AND GOAL OF STUDY}

Experimental studies have shown that intralipid (IL) is effective in reversing local anesthetic cardiac toxicity. The so-called "lipid sink" effect is suggested to be the basic mechanism of this treatment and its efficacy varies depending on the physico-chemical properties of the local anesthetics. Ropivacaine $(\mathrm{R})$ is considered a less cardiotoxic agent in comparison with other anesthetics. However, several case reports in which significant cardiac side effects have occurred after $\mathrm{R}$ administration. Limited studies have evaluated the efficacy of $\mathrm{IL}$ in the reversion of the hemodynamic effects of $R$.

\section{MATERIAL AND METHODS}

Eleven Large-White anesthetized pigs were studied. The animals were randomly assigned to two groups: control group (C-group, $n=5$ ) and intralipid group (IL-Group, $n=6$ ). A 5-French catheter (PiCCO, Pulsion Medical Systems AG) was inserted through the femoral artery (FA) to record mean aortic pressure, cardiac output (measured by transpulmonary thermodilution), LVdP/dtmax, and systemic vascular resistance index. After instrumentation and monitorization, a $R$ bolus of 5 mg.kg-1 was administered. Hemodynamic data: Heart rate (HR); mean arterial pressure (MAP); cardiac index $(\mathrm{Cl})$; maximal first derivative of left ventricular pressure ( $\mathrm{LVdP} / \mathrm{dtmax})$ and systemic vascular resistance index (SVRI) were measured at baseline, after $R$ administration and at 1, 5, 15 and $30 \mathrm{~min}$ after IL (1.5 mL/kg followed by an infusion of $0.25 \mathrm{~mL} / \mathrm{kg} / \mathrm{min})$. In C-group a saline infusion was administered instead of IL. Statistical analysis: Mann-Whitney test and Wilcoxon test as appropriate.

\section{RESULTS}

Plasma $R$ levels ranged between 7,940 to $5,450 \mathrm{ng} / \mathrm{dl}$ from 5 to $30 \mathrm{~min}$ respectively. Ropivacaine induced a significant decrease in $\operatorname{MAP}(\Delta 13 \%), \mathrm{Cl}(\Delta 28 \%), \mathrm{LVdP} / \mathrm{dtmax}(\Delta$ $41 \%)$ and in SVRI $(\Delta 16 \%)$ without significant differences between groups. There were no significant differences between lipid infusión and saline in the recovery of the hemodynamic parameters previously altered by ropivacaine through any timepoint of the experiment.

\section{CONCLUSIONS}

In the present study the lipid emulsion did not enhance hemodynamic recovery in pigs intoxicated with ropivacaine. More research is warranted to define the role of intralipid on ropivacaine cardiac toxicity.

\section{Cardiac Index}

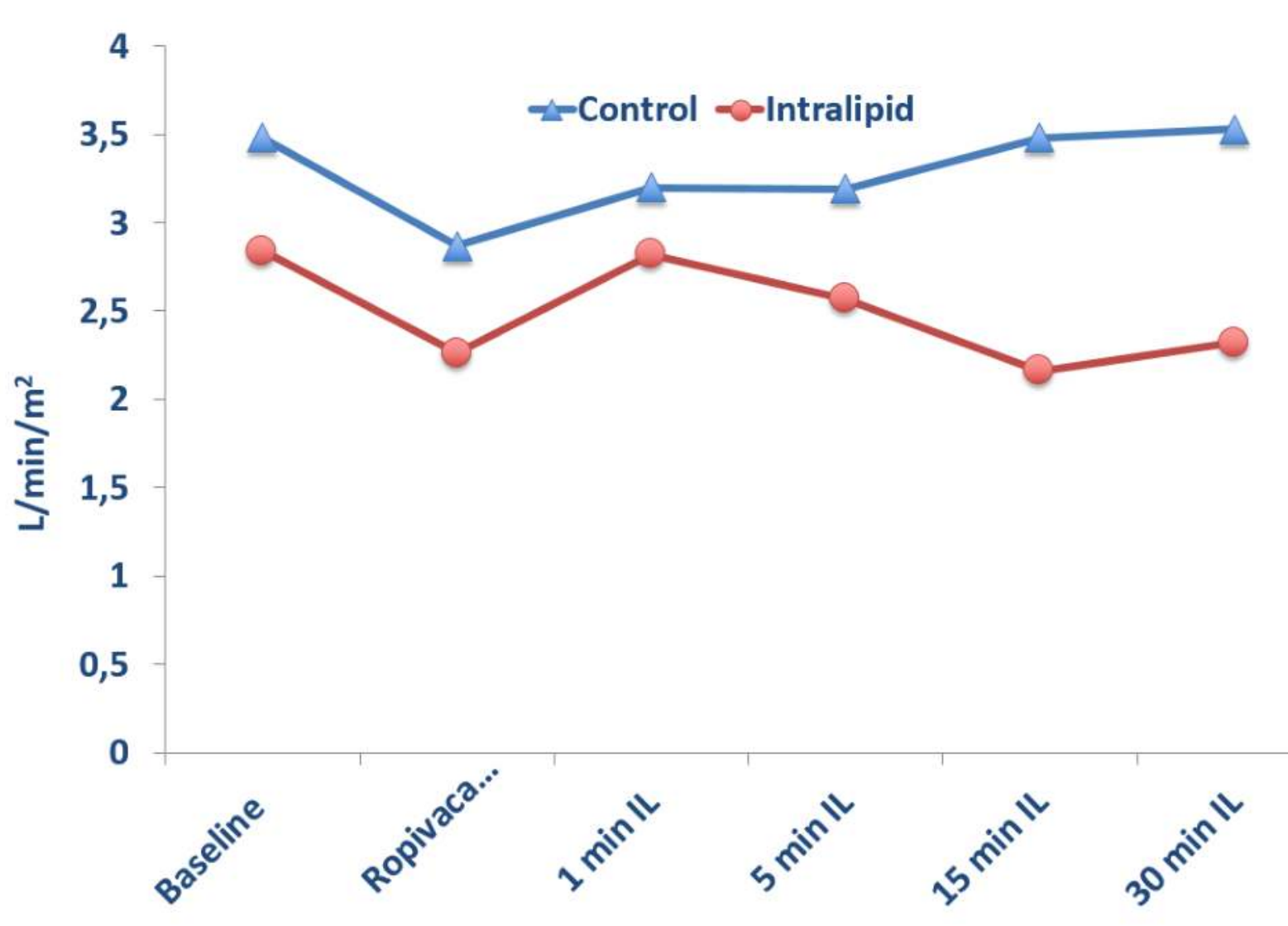

Figure 1: Diferences betwen cardiac index of intralipid group (red) and control group (blue). No significant diferences was found. 